\title{
A ACEITAÇÃO DA DISCIPLINA DE SISTEMAS DE INFORMAÇÃO CONTÁBIL: UM ESTUDO SOBRE A ATITUDE DOS ESTUDANTES EM RELAÇÃO AO USO DO COMPUTADOR
}

\section{THE ACCEPTANCE OF THE ACCOUNTING INFORMATION SYSTEMS DISCIPLINE: A STUDY ON STUDENTS ATTITUDE RELATING TO COMPUTER USE}

0 trabalho participou do XI Seminário ANPCONT promovido em Belo Horizonte (MG) nos período de 3 a 6 de junho de 2017

\section{RESUMO}

Este estudo objetivou analisar o efeito existente entre as variáveis de atitudes computacionais e a percepção de utilidade dos sistemas de informação no trabalho sobre a aceitação da disciplina de Sistema de Informação Contábil (SIC). A pesquisa foi realizada por meio de uma survey aplicada a 128 alunos matriculados a partir do quinto período do curso de Ciências Contábeis de uma universidade situada em Balneário Camboriú (SC). A análise dos dados contou com a Modelagem de Equações Estruturais via software SmartPLS 3.0. Os resultados confirmaram que as atitudes em relação aos computadores - ansiedade computacional (CA), gosto pelo uso do computador (CL), utilidade percebida do computador no local de trabalho (CUW) e importância percebida do computador no local de trabalho (CIW), exercem um efeito positivo direto sobre a aceitação pelos alunos da disciplina de Sistemas de Informação. Em contrapartida, não foram encontrados resultados significativos que sustentem o efeito percepção sobre utilidade dos SICs no trabalho e a aceitação da disciplina de Sistema de Informação Contábil. Diante desse achado, acredita-se que pesquisas posteriores podem aprofundar as análises acerca de possíveis carências da disciplina de SIC para atender as reais necessidades empresariais.

Palavras chave: Sistema de Informação Contábil. Atitudes computacionais. Utilidade dos Sistemas de Informação. Ciências Contábeis.

\begin{abstract}
This study aimed at analyzing the effect of computational attitudes variables and the perception of information systems usefulness in the work on the acceptance of the Accounting Information System (SIC) discipline. The research was carried out through a survey applied to 128 students enrolled from the fifth period of the Accounting Sciences course in a university located in Balneário Camboriú - SC. The data analysis used the Modeling of Structural Equations via software SmartPLS 3.0. The results confirmed that attitudes related to computer-computer anxiety (CA), computer use like (CL), perceived computer utility in the workplace (CUW) and perceived importance of the computer in the workplace (CIW) have a direct positive effect on students acceptance of Information Systems discipline. On the other hand, we did not find significant results that support the perception effect on SIC usefulness in the work and the acceptance of the Accounting Information System discipline. Given this finding, it is believed that further research can deepen the analysis about possible deficiencies of the SIC discipline in order to meet the business real needs.
\end{abstract}

Keywords: Accounting Information System. Computational Attitudes. Usefulness of Information Systems. Accounting Sciences.

\begin{abstract}
Marcielle Anzilago
Mestre em Contabilidade pela Universidade Federal do Paraná (UFPR). Graduada em Ciências Contábeis pela Universidade Estadual de Mato Grosso do Sul (UEMS). Doutoranda em Contabilidade pela Universidade Federal de Santa Catarina (UFSC). Contato: Centro Socioeconômico, Bloco F, Campus Universitário, Trindade Florianópolis, Santa Catarina. CEP: 88040.970 E-mail:marcianzilago@gmail.com.
\end{abstract}

\section{Franciele do Prado Daciê}

Mestre em Contabilidade pela Universidade Federal do Paraná (UFPR). Graduada em Ciências Contábeis pela Universidade Estadual de Maringá (UEM). Docente da Universidade Estadual de Maringá (UEM). Contato: Rua Afonso Pena, 317, Zona 1, Cianorte, PR. CEP: 87200.000 E-mail:frandacie@gmail.com.

\section{Juliana Andressa Negri}

Mestre em Ciências Contábeis e Atuariais pela Pontifícia Universidade Católica de São Paulo (PUC/SP). Graduada em Ciências Contábeis pela Universidade Estadual de Maringá (UEM) Docente da Faculdade Avantis. Contato: Av. Marginal Leste, 3600, Estados, Balneário Camboriú, Santa Catarina. CEP: 88339.125. E-mail: juliananegri@hotmail.com 


\section{INTRODUÇÃO}

O rápido desenvolvimento tecnológico desencadeou, essencialmente nas últimas décadas, um processo de grandes mudanças no mundo dos negócios. As transações antes registradas manualmente a fim de produzir demonstrações financeiras passaram a incorporar o uso da tecnologia, viabilizando um sistema de informações contábeis que registra e processa atividades, gerando relatórios direcionadores aos tomadores de decisão e às diversas partes interessadas (WONG; WONG, 2017). Como consequência, o nivelamento empresarial necessita de um desenvolvimento educacional constante e da formação de indivíduos dotados de capacidade de lidar com o atual ambiente de negócios. O assunto tratado mostra-se complexo e de grande preocupação em vários países, fomentando nas instituições de ensino a busca contínua pelo equilíbrio entre os objetivos educacionais e a disponibilidade de recursos muitas vezes defasados tecnologicamente (BAHAMONDEZ; WINKLER; SCHMIDT, 2011).

Diante desse cenário, sabe-se que os cursos de graduação firmam-se na estratégia de influenciar substancialmente o conhecimento dos alunos, sobretudo nos temas voltados ao nível geral e introdutório, fomentando habilidades para prosseguir na carreira de negócios (SHNEIDERMAN, 1979; EASON; DAMODARAN, 1981; FERGUSON; NEVELL, 1996). Sob essa perspectiva, observa-se que muitos órgãos de ensino reconfiguram suas grades curriculares e passam a ofertar disciplinas relacionadas ao uso de sistemas de informação na área (WONG; WONG, 2017). Entre esses campos, entende-se que o domínio do uso da tecnologia da informação (TI) está bastante voltado aos profissionais das ciências contábeis, uma vez que estes trabalham com a oferta de informações sob o lapso temporal (DAIGLE; MORRIS, 2006). Diante do exposto, a especialização no uso da TI é tratada como uma alternativa relevante para os graduados em contabilidade, especialmente para ambientes de negócios globais e dinâmicos (HOFFMAN, 2004; DARAYSEH; WAPLES; KHALEDI, 2008).

Embora a discussão sobre a integração da TI no ensino seja tratada atualmente (HOFFMAN, 2004; DARAYSEH; WAPLES; KHALEDI, 2008; BAHAMONDEZ; WINKLER; SCHMIDT, 2011), os órgãos profissionais contábeis reconhecem há tempos a necessidade de habilidades na manipulação e uso da tecnologia da informação para os que atuam nessa carreira (AMERICAN ACCOUNTING ASSOCIATION (AAA), 1988). Este reconhecimento tem crescido notoriamente devido à proliferação das tecnologias no ambiente de negócios. Nesse sentido, entende-se que as disciplinas que tratam de sistemas de informações contábeis propiciam aptidões voltadas à 'sobrevivência' nessa profissão. No entanto, mesmo sendo aplicadas as ações voltadas ao desenvolvimento do conhecimento na área da TI, não foram encontradas pesquisas que investiguem os efeitos diretos da disciplina de Sistemas de Informação Contábil sobre a atitude dos alunos em relação ao uso dos computadores.

Em face do apresentado, entende-se que a análise do acoplamento de relações comportamentais com ênfase na profissão contábil pode trazer resultados interessantes às instituições de ensino que adotam a disciplina na grade curricular, aos indivíduos que têm o interesse pela carreira, como também para divulgação à comunidade empresarial sobre o feedback da disciplina trabalhada nos cursos. Isso posto, esta pesquisa busca responder à seguinte questão: Qual a influência entre as atitudes dos alunos em relação ao uso de computadores e a utilidade percebida em relação aos sistemas de informação sobre a aceitação da disciplina de sistema de informação contábil? Ademais, a pesquisa mensura a atitude dos alunos (atitude computacional) a partir de quatro elementos: (1) a ansiedade pelo uso do computador; (2) a utilidade do computador; (3) os benefícios do seu uso; e (4) as necessidades da aplicação do computador no local do trabalho.

A relevância do tema proposto é sustentada há tempos. Em 1987, o Comitê das Regiões da Associação Americana de Contabilidade (AAA) apresentou recomendações relativas ao conteúdo de informática na contabilidade. No documento formulou-se uma abordagem para o ensino de sistemas de informação contábil, sendo atualizada conforme a recomendação das Diretrizes Internacionais de Educação para Contadores Profissionais (IFAC) sobre a competência no currículo de contabilidade. A IFAC forneceu também instruções práticas sobre o conteúdo do currículo, que consiste na capacidade de usar a planilha de processamento de texto, o banco de dados e programas de contabilidade. Além disso, estudos reconhecem o impacto da tecnologia nas empresas e sua capacidade de auxiliar no processo de tomada de decisões empresariais. A tecnologia da informação, segundo o AICPA (2005), é uma das competências funcionais e pessoais a serem desenvolvidas pelos profissionais de contabilidade no futuro.

Conforme tratado, a escassez de estudos empíricos sobre a temática abordada gera uma lacuna para pesquisas que desejam analisar a relação existente entre a aceitação da disciplina e a percepção de sua aplicabilidade. Além disso, investigações sobre o ensino de sistemas de informação em contabilidade revelam que os alunos consideram que a literatura atual não é prescritiva acerca do conhecimento e das habilidades de TI que deveriam ser assimiladas, tornando alguns tópicos da disciplina irrelevantes no curso (TAN; GODFREY, 2012).

Ademais, a justificativa para explorar a problemática respalda-se no pressuposto de que princípios organizacionais podem gerar efeitos distintos sobre a percepção dos alunos em relação a uma disciplina. Desse modo, o resultado desse estudo pode ser comparado com pesquisas realizadas em contextos distintos. Além disso, a aplicabilidade do modelo de análise proposto contribui para um maior entendimento das relações e efeitos a partir dos pressupostos teóricos, suscitando o aprofundamento sobre o tema. No âmbito acadêmico e social, o estudo contribui como feedback para avaliação do programa da disciplina e para o incentivo à adoção da temática nos currículos das universidades/faculdades que disponibilizam o curso de ciências contábeis. 


\section{REFERENCIAL TEÓRICO}

\subsection{O conteúdo do curso do Sistema de Informação Contábil e Estudos Relacionados}

As primeiras orientações direcionadas à necessidade de aprimoramento da habilidade de uso da tecnologia da informação no exercício da profissão contábil são realizadas pelo Comitê da American Accounting Association (AAA) em 1987. Diante de atividades que envolviam o uso de planilhas de processamento de texto, banco de dados e de softwares, entende-se que a ação tinha como objetivo nivelar o cenário em desenvolvimento com as competências abordadas nas Diretrizes Internacionais de Educação para Contadores Profissionais (IFAC), que já estabeleciam instruções práticas sobre o conteúdo de TI no currículo da área das ciências contábeis. Face a esse cenário, a reformulação das grades curriculares dos cursos de graduação foi necessária, tornando a oferta da disciplina de cunho obrigatório (RESOLUÇÃO nº 03/1992).

A disciplina de sistemas de informação contábil é uma matéria única e segmentada em duas vertentes complementares, um tópico sobre TI e outro quanto ao seu uso na contabilidade. Embora as abordagens busquem convergir à utilidade dessas ferramentas na profissão, percebe-se que muitas vezes a disciplina é considerada de difícil entendimento, isto porque se trata de assuntos não estruturados/quantificados e não existe um consenso quanto ao seu devido enfoque (PRADO, GUEDES e PAIVA, 2008). A questão do curso de SIC (Sistemas de Informação Contábil) e os seus métodos de ensino são investigados há tempos. Seminalmente, Wu (1983) criou um modelo de ensino geral para esses cursos, no entanto, carências empresariais induziram pesquisadores a estudar possíveis inovações nas técnicas de ensino (WU, 1986; TOGO, 1991; TOGO; MCNAMEE, 1995; MAHONEY; WELCH, 2002; STANLEY; EDWARDS, 2005; HACKBARTH; DOW; JANVRIN, 2010; MACUR, 2011; TOGO; YUTHAS, 2011).

Diante do apresentado, percebe-se que a presença de habilidades computacionais é atualmente um pré-requisito para cursos de contabilidade. Por outro lado, mesmo com a necessidade frequente de desenvolvimento do aprendizado, observa-se que nem todos os materiais (livros de suporte ao estudo) aderem à ideia de propor livros didáticos com tarefas multidisciplinares e que integrem tópicos encontrados em vários capítulos. Em consequência, cabe aos professores desenvolver seus próprios projetos para facilitar a aprendizagem de conceitos vinculados à disciplina (WU, 1986; TOGO, 1991).

Wu (1986) e Togo (1991) relatam experiências com projetos de TI na contabilidade e sugerem a adoção de metodologias que vinculam conceitos computacionais e de gestão a fim de aumentar o aprendizado. O ciclo contábil (WU, 1986) e o orçamento-mestre (TOGO, 1991) são exemplos de tópicos integrativos adequados para o uso de computadores. Até mesmo tarefas de contabilidade complexas, como definição de planos de benefícios (KACHELMEIER, JONES e KELLER, 1992), podem ser aplicadas ao uso do computador, uma vez que exigem que o aluno inter-relacione elementos-chave do curso.

Na década de 2000, diante de diversas introduções tecnológicas, as pesquisas passam a abordar diferentes formas de introdução da TI no ensino. Mahoney e Welch (2002) sugerem o uso de filmes para integrar a TI como conteúdo do curso. Já Stanley e Edwards (2005) indicam a abordagem de SIC por meio de materiais multimídias (CD-ROM) que relacionem o ciclo de transações de acordo e o ambiente real de negócios. Hackbarth, Dow e Janvrin (2010) indicam o emprego do método de ensino computadorizado em sala de aula, apontando que o desempenho dos alunos nos cursos SIC torna-se mais representativo se comparado ao método de ensino tradicional. Togo e Yuthas (2011), por sua vez, sugerem uma forma de ensino cooperativo em sistemas de role-play sobre organização, em quem se apresentam diferentes ciclos de transação em operações de negócios. Por fim, Macur (2011) propôs um formato de ensino via laboratório com cases que abordam a prática contábil de análise e escrituração aplicada por meio de softwares de contabilidade.

Conforme observado, estudos realizados até a década de 2000 voltam-se predominantemente à análise do emprego de metodologias tecnológicas no ensino. No entanto, após esse período, as abordagens adotam uma nova roupagem quanto aos SICs, objetivando então mensurar a atitude dos alunos em relação ao computador. Darayseh, Waples e Khaledi (2008), por exemplo, ao verificarem a importância que graduandos davam ao conhecimento de TI sobre o sucesso na carreira profissional, mostraram que a temática era considerada como relevante no currículo de contabilidade. Adicionalmente, a abordagem dada por Morris e Daigle (2004) mostrou como se comporta a atitude do aluno em relação ao computador antes e depois de ter se submetido ao curso SIC. O resultado das observações indicou que uma atitude positiva (proativa) em relação ao computador exerceu mais influências no curso SIC, ou seja, experiências nesse ambiente possibilitam ao aluno compreender melhor o conceito SIC. Além das pesquisas supramencionadas, outros estudiosos também investigaram o uso de ferramentas específicas de SIC, como o software Enterprise Resource Planning (ERP) e o aprendizado de serviços em cursos de SIC (GUJARATHI, 2005).

\subsection{Atitude em relação ao computador, utilidade do sistema de informação contábil no local de trabalho e aceitação do curso Sistema de Informação Contábil}

Conforme a Teoria da Ação Racional, a atitude é algo que determina o comportamento de um indivíduo (AJZEN, 1991), sendo uma combinação de confiança, do sentimento de uma pessoa em relação a um objeto e da tendência a agir em relação a ele (ao objeto). Dessa forma, a atitude em relação ao computador vincula-se ao envolvimento do indivíduo para com o uso do computador. Essas ações, por sua vez, encontram-se comumente atreladas aos sentimentos de ansiedade, confiança, apego e utilidade (LOYD; LOYD, 1985). 
Estudos evidenciam que as atitudes afetivas no que se refere ao uso de computadores são fortemente motivadas por dois elementos: as práticas de alfabetização e a consciência e competência para usá-los. Borthick e Clark (1987) relatam que a atitude do estudante quanto à integração deste elemento é uma proxy de percepção do valor da aprendizagem a partir do uso de computadores. Desse modo, uma ação proativa estaria relacionada à receptividade e expectativas que o indivíduo percebe com o seu uso no aprendizado. Minch e Ray (1986) argumentam que o grau de ansiedade, o medo, a aversão ao uso de instrumentos tecnológicos e a 'ansiedade informática' (sentimento causado pela falta de familiaridade com o elemento) podem causar sentimentos representativos na aprendizagem dos alunos.

Na área contábil, acredita-se na relação de sucesso que as habilidades de TI podem proporcionar aos profissionais (ELLIOT, 1997). Exemplos dessa necessidade de aptidão podem ser observados em diversos exames de ordem, como o ANBIMA, Professional Certification (CPA), o Certified Management Accountant (CMA) e o Certified in Financial Management (CFM) (AICPA, 2004). Nesse sentido, a relevância de investigações que exploram o impacto das disciplinas de SIC sobre a atitude computacional dos alunos respalda-se na análise dos efeitos que esse elemento exerce sobre o desempenho e a satisfação no trabalho. Pesquisas relatam que a melhoria nas atitudes dos alunos é atingida a partir do momento em que fazem um curso introdutório de informática (OMAR, 1991; HARRIS, 1992), outras defendem que à medida que o indivíduo adquire experiência quanto ao uso do computador, sua percepção torna-se mais positiva quanto a ele (TORKZADEH; KOUFTEROS, 1993). Diante desses achados e de um ambiente que se movimenta no âmbito digital, compreende-se que essa discussão sobre atitudes afetivas e a percepção de utilidade não estejam somente atreladas ao uso do computador em si, mas ao uso de sistemas informacionais que, sobretudo, estão vinculados à utilização da tecnologia. Ademais, embora exista a expectativa de que a exposição ao computador melhoraria as atitudes dos alunos, Ravel (1991) e Dunn e Grabski (1998) evidenciam que os estudantes da área contábil tendem a ter atitudes desfavoráveis em relação à disciplina de Sistema de Informação Contábil, especialmente quando inseridos em cursos de contabilidade com grades curriculares tradicionais. Em ambos os estudos evidencia-se a preocupação em relação aos achados, uma vez que o conteúdo de SIC tipicamente enfatiza conceitos bastante abstratos, tais como sistemas, processos, controles e design. O fato depara-se com o perfil de procedimentos objetivos, normativos e precisos adotados pela maioria dos cursos de contabilidade. Frente a esse cenário, advertências são realizadas quanto à possibilidade de frustração e alienação da disciplina diante do foco do curso (RAVEL, 1991; DUNN; GRABSKI, 1998).

\section{PROCEDIMENTOS METODOLÓGICOS}

\subsection{Hipóteses testadas e modelo estrutural teórico do estudo}

A abordagem tratada em Morris e Daigle (2004) e Darayseh, Waples e Khaledi (2008) evidencia um campo bastante interessante para exploração - a percepção da utilidade do Sistema de Informação Contábil no local de trabalho, sobretudo quando aliado à atual fase do 'mundo digital'. O estudo de Weli (2015) respalda-se em Darayseh, Waples e Khaledi (2008) e propõe três hipóteses que verificam os efeitos que as variáveis atitude computacional e percepção do aluno sobre a utilidade da disciplina de SIC exercem sobre a aceitação do conteúdo abordado nesse curso.

A fim de medir a atitude para com o computador, Weli (2015) propõe quatro variáveis: (1) a ansiedade computacional (ansiedade por usar o computador), (2) o gosto pelo uso do computador, (3) a percepção de utilidade do computador no local de trabalho e a (4) percepção da importância do computador no local de trabalho. A variável externa utilidade percebida do SIC refere-se à importância que o conteúdo de SIC possui para influenciar as atividades no local de trabalho. Por fim, a aceitação da disciplina SIC mensura a percepção do aluno sobre a importância do tema e material fornecido no curso. A Figura 1 apresenta o modelo acima discutido:

Figura 1 - Modelo estrutural teórico

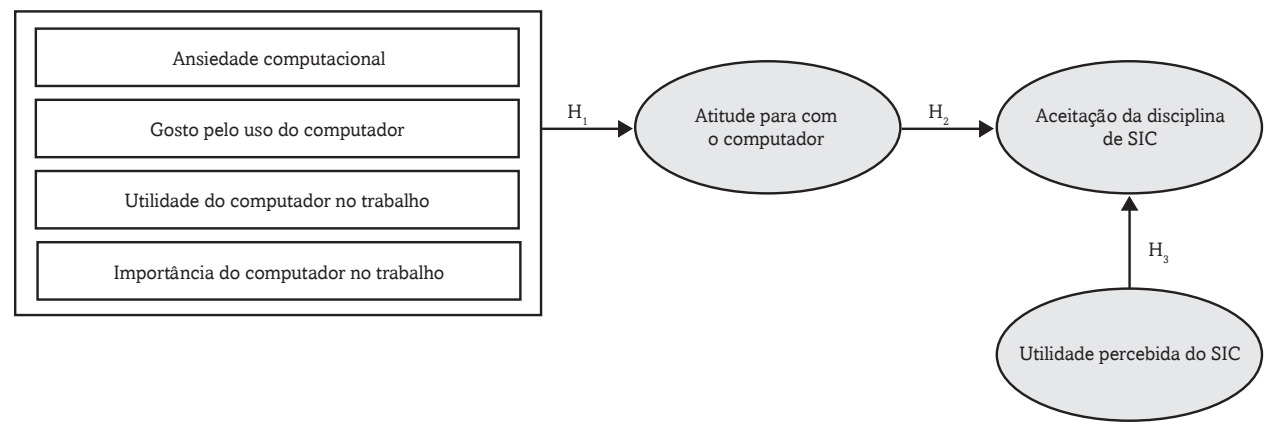

Uma vez definido o modelo teórico, a influência entre as atitudes afetivas dos alunos e a utilidade percebida em relação aos SICs sobre a aceitação dessa disciplina no curso de Ciências Contábeis foi analisada a partir da reaplicação dos procedimentos adotados por Weli (2015). Convém relatar que o estudo de Weli (2015) realizou-se em Jakarta (Indonésia), desse modo, algumas terminologias e abordagens foram adaptadas para o entendimento do contexto em cenário local. Assim, as hipóteses aplicadas na pesquisa são: 
H1: A ansiedade computacional, o gosto pelo uso do computador, a utilidade computacional percebida no local de trabalho e a importância do computador no local de trabalho influenciam a atitude do estudante em relação ao computador.

H2: A atitude do aluno em relação ao computador está positivamente relacionada com a aceitação do Sistema de Informação Contábil.

H3: A percepção de utilidade da disciplina de Sistema de Informação Contábil no local de trabalho é positivamente relacionada com a aceitação da disciplina de Sistema de Informação Contábil pelos alunos.

\subsection{Classificação da pesquisa e procedimento de análise dos dados}

Esta pesquisa adota uma abordagem quantitativa em relação ao problema e aplica a estratégia de survey para coleta dos dados. A população do estudo compreendeu 150 alunos regularmente matriculados entre o quinto e oitavo período (semestre) do curso de Ciências Contábeis de uma instituição de ensino superior (IES) situada em Balneário Camboriú (SC). Convém relatar que, para a seleção da amostra, utilizou-se como requisito a participação (atual ou passada) dos discentes na disciplina de Sistemas de Informação Contábil (ofertada pela IES no quinto período).

Os objetivos e motivações da pesquisa foram apresentados aos respondentes presencialmente, e a coleta dos dados realizou-se nos meses de fevereiro e março do ano de 2016 por meio da aplicação do questionário aos alunos presentes em sala na data da visita. Assim, dos 150 alunos matriculados, obteve-se um total de 128 respostas válidas - valor amostral correspondente a $85 \%$ da população. Ademais, o instrumento aplicado na pesquisa foi adaptado de Morris e Daigle (2004) e Darayseh, Waples e Khaledi (2008) e constituído por 51 questões, 4 relativas a variáveis demográficas e as demais relacionadas à Ansiedade Computacional (CA), ao Gosto pelo Uso do Computador (CL), à Utilidade do Computador no Local de Trabalho (CUW), Importância do Computador percebida no local de Trabalho (CIW), Aceitação da disciplina de SIC (PNM) e Utilidade percebida dos SICs (AUW). As questões foram formatadas em escala tipo likert (discordo totalmente/concordo totalmente). As distribuições das questões às suas respectivas variáveis são apresentadas na Tabela 1.

Tabela 1 - Variáveis do questionário

\begin{tabular}{llc}
\hline Variável & Bloco: Descrição das variáveis & Quantidade de questões \\
\hline CA & Ansiedade computacional & 4 \\
CL & Gosto pelo uso do computador & 4 \\
CUW & Utilidade do computador no local de trabalho & 5 \\
CIW & Importância do computador percebida no local de trabalho & 21 \\
PNM & Aceitação da disciplina de Sistema de Informação Contábil & 5 \\
AUW & Utilidade percebida dos Sistemas de Informações Contábeis
\end{tabular}

Fonte: Autores (2017)

Convém ressaltar que alguns cuidados foram adotados durante a tradução do instrumento de pesquisa. A fim de viabilizar a abordagem no estudo, a versão vernácula sofreu algumas adaptações quanto aos termos empregados na denominação dos construtos. Além disso, para atender ao critério de validade de expressão do questionário, algumas palavras foram ajustadas à realidade vivida pela população. Por fim, após a tradução das assertivas, foi feito um teste piloto com profissionais da área de modo a confirmar a compreensibilidade das frases.

A análise dos dados contou com a aplicação de modelagem de equações estruturais (SEM) via software SmartPLS e Statistical Package for the Social Science (SPSS). Conforme Bido et al. (2010), o Partial Least Squares Structural Equation Modeling (PLS-SEM) é uma técnica de estimação de regressão linear que se baseia na decomposição de matrizes de variáveis e de covariáveis a fim de realizar a validação e adequação do modelo. Além disso, a utilidade do PLS torna-se relevante à medida que testa hipóteses com dados mínimos, sendo especialmente interessante para amostras de dimensões pequenas (HALL, 2008).

Acerca da representatividade amostral, Chin e Newsted (1999) orientam que para aplicar a modelagem em PLS há como requisito uma amostra mínima de pelo menos dez vezes a regressão múltipla que contém o maior número de variáveis do modelo. Nesse sentido, a amplitude amostral atende às expectativas, uma vez que a regressão com maior número de variáveis refere-se ao construto de atitude para com o computador - possuindo quatro variáveis. Além disso, Hair Jr., Hult, Ringle e Sarstedt (2014) indicam a realização de uma análise do poder estatístico a partir do software $G^{\star}$ Power. A estimação considerou como parâmetros (1) o poder do teste $=0,95$, (2) tamanho do efeito (f2) $=0,15$, (3) maior número de preditores para variável $=4$ (variável atitude para com o computador). Os resultados apontaram um tamanho mínimo amostral de 74 respondentes, validando então a etapa de coleta de dados. 


\section{ANÁLISE DOS DADOS \\ 4.1 Perfil dos respondentes}

O primeiro bloco do instrumento da pesquisa mapeou o perfil dos respondentes em quatro principais características que possibilitaram avaliar o comportamento amostral. Desse modo, a Tabela 2 apresenta as seguintes categorias da amostra: gênero, idade, período do curso no qual os alunos estão matriculados e área de atuação (trabalho).

Tabela 2 - Perfil dos respondentes

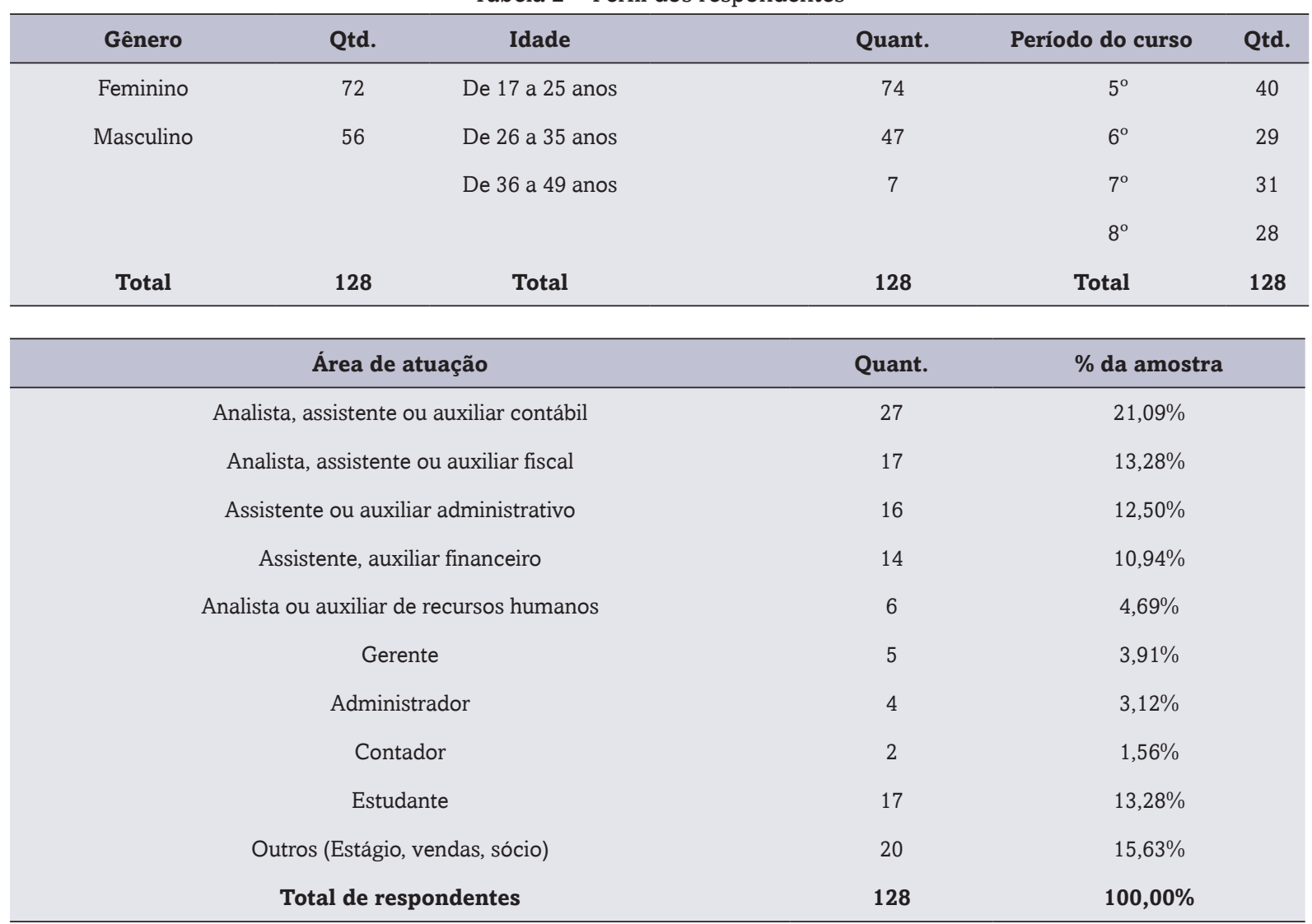

Fonte: Dados da pesquisa

Conforme disposto na Tabela 2, entre os 128 participantes da pesquisa, 56,25\% são do gênero feminino e $43,75 \%$ masculino. A idade varia entre 17 e 49 anos, sendo aproximadamente dois terços (57,81\%) dos respondentes compreendidos entre 17 e 25 anos. Ademais, 36,72\% da amostra possuem entre 26 a 35 anos, e 5,47\% enquadram-se entre 36 e 49 anos. Com relação ao período de curso em Ciências Contábeis, percebe-se uma maior concentração de alunos matriculados no quinto período (31,25\%), seguidos pelo sétimo $(24,22 \%)$, sexto $(22,65 \%)$ e oitavo período $(21,88 \%)$. Por fim, a abordagem sobre a atividade de atuação teve como intenção verificar se as atividades exercidas pelos discentes eram condizentes com o curso e, consequentemente, com o alvo esperado para esta pesquisa (pessoas atuantes da área contábil). Entre as atividades mapeadas, as mais representativas somam $57,85 \%$ da amostra, sendo respectivamente divididas em setor contábil $(21,09 \%)$, fiscal $(13,28 \%)$, administrativo $(12,50 \%)$, financeiro $(10,94 \%)$. Alguns dos respondentes relataram também somente estudar $(13,28 \%)$, situação que possivelmente acarreta baixa inserção em situações que exploram os sistemas de informação. Além dessas categorias, uma pequena parcela dos respondentes atua como gerente, administrador e contador. A partir desses apontamentos, acredita-se que a maior parte dos respondentes é apta a posicionar-se acerca da utilidade do sistema de informação contábil no ambiente de trabalho. Após a análise da homogeneidade amostral, a próxima seção descreve o modelo estrutural proposto.

As relações encontradas a partir da técnica PLS para o uso de modelagem de equações estruturais são observadas por meio da Figura 2, que organiza e denomina os construtos como: (1) utilidade percebida dos Sistemas de Informações Contábeis (AUW), (2) ansiedade computacional (CA), (3) importância percebida do computador no local de trabalho (CIW), (4) gosto pelo uso do computador (CL), (5) utilidade do computador no local de trabalho (CUW) e (6) aceitação do Sistema de Informação Contábil (PNM). A fim de legitimar o modelo, adotou-se como procedimento preliminar a análise fatorial exploratória, que atestou a validade dos indicadores utilizados para medição dos construtos. 
Figura 2 - Modelo empírico - Equações estruturais

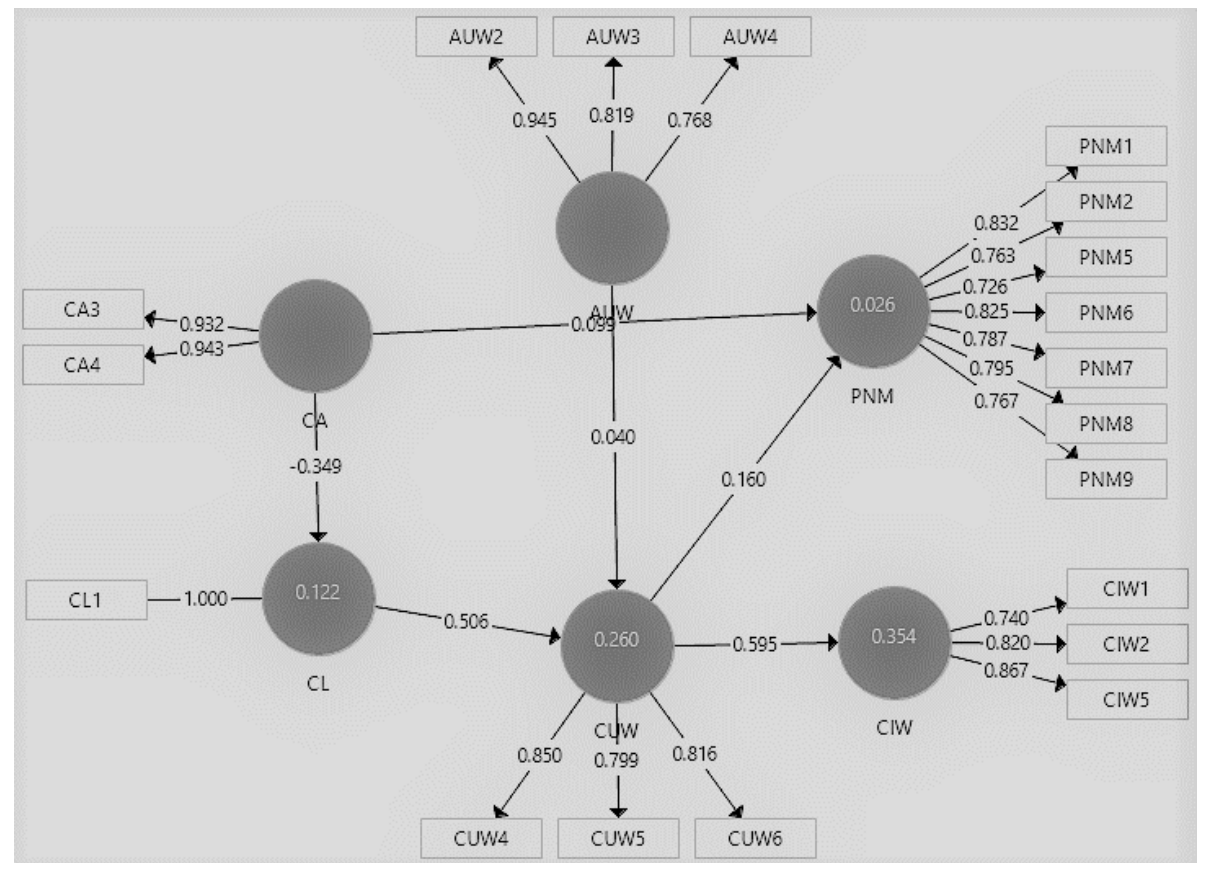

Fonte: Dados da pesquisa

Semelhante aos procedimentos adotados por Weli (2015), as variáveis ansiedade computacional (CA) e gosto pelo uso do computador (CL) foram decompostas por meio de uma análise fatorial confirmatória (AFC). A técnica aplicada direcionou as respostas obtidas para as assertivas de CA e CL de forma a gerar um só fator, ou seja, mensurar uma só variável. Logo, o agrupamento realizado entre CA e CL as torna uma só variável de mensuração para o construto de atitude para com o uso do computador e que, por sua vez, relaciona-se com a aceitação da disciplina de SIC. Ao realizar tal procedimento, o autor defende que a ansiedade e a apreciação pelo uso do computador podem ser agregadas como uma forma de atitude.

Quanto aos procedimentos de validação por meio da análise fatorial exploratória (AFE), dezesseis questões foram excluídas em razão da presença de comunalidades. Os valores mínimo e máximo para cada assertiva, conforme Fávero et al. (2009), podem variar entre, respectivamente, 0 e 1 . No entanto, os indicadores aceitáveis para atender aos critérios do teste devem apresentar valores superiores a 0,50 (abaixo desse valor recomenda-se excluir o indicador, desde que não interfira no modelo teórico). A fim de atender a esse requisito, as seguintes questões foram eliminadas de cada construto: Q.1 e Q.5 - Sistemas de Informações Contábeis percebidos; Q.1 e Q.2 - ansiedade computacional; Q.3 e Q.4 - Importância do computador percebida no local de trabalho; Q.2, Q.3 e Q.4 - Gosto pelo uso do computador; Q.1, Q.2, Q.3, Q.7 e Q.8 - Utilidade computada percebida no local de trabalho; e, por fim, Q.3 e Q.4 - Aceitação do Sistema de Informação Contábil. As demais variáveis obtiveram o nível mínimo exigido.

Segundo Hair Jr. et al. (2009), a confiabilidade é considerada como adequada quando as cargas fatoriais apresentam um alfa de Cronbach de pelo menos 0,70. Para pesquisas de cunho exploratório, Hair Jr. et al. (2009) relatam que são excepcionalmente aceitáveis níveis de carga a partir de 0,60. Desse modo, após a exclusão de alguns indicadores com comunalidade insatisfatória, as cargas ajustaram-se adequadamente aos requisitos para validação dos construtos e o alfa de Cronbrach. Como procedimento adicional para garantir maior robustez e confiabilidade dos indicadores, a análise fatorial confirmatória foi também aplicada a cada construto, conforme apresentado na Tabela 3.

Tabela 3 - Análise fatorial confirmatória

\begin{tabular}{cc}
\hline Variáveis & Alpha de Cronbach \\
\hline AUW & 0,849 \\
CA & 0,863 \\
CIW & 0,741 \\
CL & 0,998 \\
CUW & 0,760 \\
PNM & 0,900 \\
\hline
\end{tabular}

Fonte: Dados da pesquisa. 
Após a realização dos testes de validade e adequação do modelo, aplicam-se as técnicas necessárias para garantir-se a validade convergente (AVE) e a validade discriminante dos construtos. A AVE reflete o grau de concordância existente entre os construtos do modelo, ou seja, se pelo menos duas variáveis que utilizam diferentes métodos para formação podem ter relações entre si. Por outro lado, a validade discriminante tem como finalidade verificar o grau em que essas medidas (variáveis / construtos) se diferenciam entre si - mensuram elementos diferentes (BAGOZZI; PHILLIPS, 1982).

Hair Jr. et al. (2009) explicam que a validade convergente se refere à variância média extraída dos construtos no modelo, a qual demonstra a variância compartilhada entre os indicadores de cada variável latente. Adicionalmente, Bido (2008) relata que a validade convergente indica o grau em que os itens teoricamente deveriam estar correlacionados, sendo essa verificação realizada por meio dos valores das cargas fatoriais da AVE e da confiabilidade composta (CR). A Tabela 4 expõe os valores encontrados para AVE e CR juntamente às comunalidades e $\mathrm{R}^{2}$.

Tabela 4 - Validade e consistência dos constructos

\begin{tabular}{ccccc}
\hline Variáveis & AVE & Confiabilidade composta (CR) & $\mathbf{R}^{2}$ & Comunalidades \\
\hline AUW & 0,718 & 0,883 & 0,000 & 0,849 \\
CA & 0,879 & 0,936 & 0,000 & 0,863 \\
CIW & 0,657 & 0,851 & 0,354 & 0,741 \\
CL & 0,998 & 0,998 & 0,122 & 0,980 \\
CUW & 0,676 & 0,862 & 0,260 & 0,760 \\
PNM & 0,618 & 0,919 & 0,026 & 0,900 \\
\hline
\end{tabular}

Fonte: Dados da pesquisa

A partir desses resultados, entende-se que os valores de AVE e CR atendem ao preconizado na literatura e a validade convergente é considerada satisfatória. Tal afirmação respalda-se no atendimento de dois critérios: (1) a variância da média extraída (AVE) dos construtos latentes manifesta-se com valores superiores a 0,50 (FORNELL; LARCKER, 1981) e (2) os níveis de confiabilidade composta enquadram-se entre de 0,70 e 0,90 (BIDO, 2008). Adicionalmente, os resultados mostram que a atitude dos respondentes para com o uso do computador é determinada em sua maior parte pelas variáveis de percepção da importância do uso dos sistemas computacionais no local de trabalho $\left(C I W\right.$ : $\left.R^{2}=0,354\right)$ e da utilidade do computador no local de trabalho (CUW: $\mathrm{R}^{2}=0,260$ ). Por esses dados, depreende-se que os quesitos de validade convergente e validade composta no modelo proposto são atendidos. A próxima etapa aplicada refere-se à análise da validade discriminante e da consistência do modelo. A validade discriminante respalda-se nas orientações obtidas pelo critério de Fornell-Larcker e tem como finalidade verificar o quanto um construto é individualmente distinto dos demais. Nesse sentido, a raiz quadrada da variância média extraída (AVE) de cada construto é comparada com a correlação existente entre as variáveis latentes. Nesse caso, a validade discriminante é confirmada se as correlações entre os construtos forem menores que a raiz quadrada das AVEs (FORNELL; LARCKER, 1981). Na Tabela 5 apresentam-se os valores da correlação entre as variáveis do modelo.

Tabela 5 - Consistência e validade do modelo

\begin{tabular}{cccccccc}
\hline Variáveis & AUW & CA & CIW & CL & CUW & PNM \\
\hline AUW & $\mathbf{0 , 8 4 7}$ & - & - & - & - & - \\
CA & $-0,061$ & $\mathbf{0 , 9 3 8}$ & - & - & - & - \\
CIW & 0,146 & 0,186 & $\mathbf{0 , 8 1 1}$ & - & - & - & - \\
CL & 0,047 & 0,349 & 0,319 & $\mathbf{0 , 9 9 9}$ & - & - & - \\
CUW & 0,064 & 0,285 & 0,595 & 0,508 & $\mathbf{0 , 8 2 2}$ & - \\
PNM & 0,333 & 0,054 & 0,183 & 0,015 & 0,131 & $\mathbf{0 , 7 8 6}$ \\
\hline
\end{tabular}

Fonte: Dados da pesquisa.

Conforme Tabela 5, nenhuma das correlações entre os construtos foi superior à raiz quadrada da variância média extraída (AVE). Portanto, verifica-se a existência de validade discriminante no modelo, ou seja, as dimensões avaliadas no estudo se discriminam e confirmam o atendimento a tal quesito. Por conseguinte, a Tabela 6 evidencia a análise das relações ou caminhos (Pathcoefficients), mostrando a relação e a significância estabelecida entre os construtos (HAIR JR. et al., 2009). 
Tabela 6 - Efeitos da relação entre os construtos

\begin{tabular}{|c|c|c|c|c|c|c|c|}
\hline \multirow{2}{*}{$\begin{array}{l}\text { Relação entre os } \\
\text { construtos }\end{array}$} & \multirow[t]{2}{*}{ Hipótese } & \multicolumn{3}{|c|}{ Efeito direto } & \multicolumn{3}{|c|}{ Efeito Indireto } \\
\hline & & valor & t-value & $p$-value & valor & t-valor & p-value \\
\hline AUW $®$ CUW & - & 0,040 & 0,289 & 0,772 & - & - & - \\
\hline $\mathrm{CA} \circledast \mathrm{CL}$ & - & $-0,349$ & 3,026 & 0,002 & - & - & - \\
\hline $\mathrm{CA} \circledast \mathrm{PNM}$ & $\mathrm{H} 2$ & 0,099 & 0,623 & 0,533 & 0,028 & 1,010 & 0,313 \\
\hline CL $® \mathrm{CUW}$ & - & 0,506 & 5,823 & 0,000 & - & - & - \\
\hline CUW ® CIW & - & 0,595 & 4,963 & 0,000 & - & - & - \\
\hline CUW $®$ PNM & - & 0,160 & 1,332 & 0,184 & - & - & - \\
\hline CA® CL® CUW® CIW & $\mathrm{H} 1$ & 0,483 & 4,604 & 0,001 & 0,506 & 2,435 & $0,023 * *$ \\
\hline AUW @ CUW ® PNM & $\mathrm{H} 3$ & 0,146 & 1,289 & 0,576 & 0,595 & 4,682 & 0,105 \\
\hline
\end{tabular}

Nota: * Significante a 0,$01 ; * \star$ Significante a 0,05, *** Significante a 0,10

Fonte: Dados da pesquisa.

Os resultados dispostos na Tabela 6 mostram que, embora o modelo atenda aos critérios de adequação, os efeitos diretos propostos por Weli (2015) não foram significativos para as relações entre os construtos, com exceção dos efeitos encontrados para os caminhos de controle (CA®CL:0,002; CL $₫$ CUW:0,000 e CUW $® C I W: 0,000)$. Adicionalmente, observa-se um efeito indireto pequeno, porém significativo, para relação entre os constructos CA $\otimes C L \circledast C U W \circledast C I W$ $(0,023)$. Sob essa perspectiva, os resultados do teste permitem inferir que as atitudes em relação aos computadores - ansiedade computador (CA), gosto do computador (CL), utilidade percebida do computador no local de trabalho (CUW) e importância percebida do computador no local de trabalho (CIW) - podem exercer, de alguma forma, um efeito positivo sobre a aceitação dos alunos na disciplina de sistemas de informação.

Isso posto, observa-se que os resultados encontrados permitem afirmar que a hipótese $\mathrm{H} 1$ - que assume um efeito indireto entre os indicadores de atitude computacional - não pode ser rejeitada.

Diante da convergência com estudos datados como de Minch e Ray (1986), entende-se atualmente que fatores como o grau de 'ansiedade informática' e o receio de não adequar-se ao nivelamento organizacional/ambiente no qual o indivíduo está inserido (diante da utilidade percebida do computador) podem causar sentimentos representativos para aceitabilidade da aprendizagem dos alunos. Estudos como os de Darayseh, Waples e Khaledi (2008) sustentam também o vínculo entre conhecimento de tecnologia da informação e sucesso no trabalho, o que indica que possivelmente a percepção de utilidade permite maior aceitabilidade para aprendizagem. Ademais, ratificando o apresentado em Weli (2015), denota-se que a ansiedade pelo computador, o gosto pelo computador e sua utilidade percebida no trabalho são elementos que influenciam a relação entre aluno e computador. Esse cenário permite propor duas discussões para pesquisas futuras. Uma delas está vinculada à possível relação entre o medo e a redução da atitude proativa em relação a ele, uma vez que o indivíduo é influenciado por um sentimento de desconforto e ansiedade, ou até mesmo o sentimento de 'obstáculo'.

Em alternativa, entende-se que uma elevada percepção de utilidade dos computadores no ambiente de trabalho (em termos de atitudes em relação aos computadores) pode fomentar o interesse pela disciplina de SIC e sua aceitabilidade no ambiente de ensino. Desse modo, o maior conhecimento sobre os sistemas de informação computacional proporcionaria também facilidade de seu uso e garantiria melhores oportunidades de promoção ou enquadramento em novos empregos em relação aos demais indivíduos. Em meio à área contábil, acredita-se nessa relação de sucesso que as habilidades de TI podem gerar aos profissionais (ELLIOT, 1997). Acredita-se também que uma boa comunicação auxilia no posicionamento dos indivíduos para com os computadores, e que a percepção de sua importância e utilidade no ambiente de trabalho fortalece a atitude proativa do indivíduo em relação aos sistemas computacionais.

Os demais achados dessa pesquisa não apresentaram relações positivas e significativas entre as variáveis de atitude do aluno em relação ao computador e a aceitação da disciplina de Sistema de Informação Contábil, fato que permite rejeitar H2. Esses resultados diferem de Weli (2015), que sustenta a hipótese ao afirmar que uma boa atitude em relação ao computador exerce um bom efeito sobre as atitudes dos alunos no estudo de TI. Acredita-se que a divergência entre os cenários pode ser resultante do fator cultural (costumeiro) dos alunos com relação a usar computador em seu ambiente de trabalho, ou ainda, atribuída às restrições impostas ao seu uso.

Semelhantemente, H3 não obteve significância representativa nos dados, permitindo inferir que na amostra estudada não existem relações entre a utilidade dos SICs no local de trabalho e a aceitação da disciplina de Sistemas de Informações Contábeis pelos alunos. É importante destacar que Weli (2015) também não encontrou suporte significativo para comprovar algum efeito entre a percepção da utilidade do curso Sistemas de Informações Contábeis no local de trabalho. Como um todo, os resultados deste estudo são adequados à base da Teoria da Ação Racional (AJZEN, 1991), a qual defende que a atitude é uma das coisas que definem o comportamento de uma pessoa. Logo, acredita-se que a aceitação dos Sistemas de Informações Contábeis pelos alunos é explicada pelo nível de controle.

Os achados deste estudo assemelham-se a Darayseh, Waples e Khaledi (2008), que defendem que o conhecimento e as atitudes em relação aos computadores influenciam no currículo de contabilidade, sobretudo aqueles que se 
adequam a um perfil de contador prospectivo à carreira dos negócios. Já Morris e Daigle (2004) apontam um cenário diferente, em que a atitude positiva em relação aos computadores influenciou o curso de SIC que, por sua vez, afetou positivamente o entendimento dos alunos sobre o conceito de SIC. Diante dos resultados, os autores propuseram que o ensino de SIC deveria ser integrado com a experiência do aluno na utilização dos sistemas computacionais, além de o material SIC estar conectado ao conceito de tecnologia do computador. No estudo observou-se que os métodos de aprendizagem frequentemente aplicados em sala de aula evoluíam diante das necessidades informacionais (inicialmente palestras e vídeos, estudos de campo, seguidos pelo uso do e-learning, da internet e do Power Point).

Acerca das metodologias de inserção da tecnologia de informação no ambiente de ensino, estudos anteriores, como de Mahoney e Welch (2002), sugerem a integração da TI no curso a partir do uso de filmes. Stanley e Edwards (2005), por sua vez, indicam que a abordagem dos SICs deve ser implantada por meio de materiais multimídias (CD-ROM) que relacionem o ciclo de transações de acordo e o ambiente real de negócios. Hackbarth, Dow e Janvrin (2010) indicam o emprego do método de ensino computadorizado em sala de aula, apontando que o desempenho dos alunos nos cursos SIC torna-se mais representativo se comparado ao método de ensino tradicional. Nesse sentido, acredita-se que promover a convivência com o ambiente técnico por meio da aplicação do registro das operações contábeis em softwares (gratuitos, versão demonstração) e a reflexão sobre as inter-relações entre módulos e a análise de cenários empresariais por meio da geração de relatórios pode contribuir potencialmente para o interesse no conteúdo programático da disciplina. Adicionalmente, Togo e Yuthas (2011) sugerem uma forma de ensino cooperativo em sistemas de role-play sobre organização, em que se apresentam diferentes ciclos de transação em operações de negócios. Há também a proposta de formatos de ensino via laboratório, com cases que abordam a prática contábil de análise, e escrituração aplicada por meio de softwares de contabilidade, conforme indicado por Macur (2011).

Diante dos resultados encontrados na presente pesquisa, acredita-se na possibilidade de que as abordagens da atual grade da disciplina SIC estejam defasadas diante das necessidades de conhecimento no ambiente de trabalho. Este e outros aspectos, como metodologia de ensino, inserção de temas atuais e específicos, assim como a aplicabilidade de estudos de campo, podem ser alternativas viáveis para valorizar a disciplina.

\section{CONSIDERAÇÕES FINAIS}

Este estudo objetivou analisar o efeito existente entre duas variáveis - (1) as atitudes computacionais dos alunos e (2) a percepção que os alunos possuem em relação à utilidade dos sistemas de informação - sobre a variável (3) de aceitação da disciplina de sistema de informação no curso de Ciências Contábeis. A partir desse cenário, o estudo defende a ideia de que quanto maior for o medo de uma pessoa em relação ao uso do computador (ou tecnologias afins), menor será sua atitude proativa em relação ao seu uso. Entende-se por medo sentimentos que possam gerar desconforto ou até mesmo aversões ao uso dessa tecnologia.

O desenvolvimento da pesquisa realizou-se por meio de survey aplicado junto a 128 alunos de uma IES situada em Balneário Camboriú (SC). A coleta dos dados contou com o instrumento de pesquisa do estudo de Morris e Daigle (2004) e Darayseh, Waples e Khaledi (2008) e da reaplicação das hipóteses apresentadas por Weli (2015). Os achados confirmaram que as atitudes em relação aos computadores - ansiedade pelo uso do computador (CA), gosto pelo computador (CL), utilidade percebida do computador no local de trabalho (CUW) e a importância percebida do computador no local de trabalho (CIW) - exercem um efeito positivo sobre a aceitação dos alunos na disciplina de Sistemas de Informação Contábil.

Acerca dessas variáveis, CUW e CIW foram as mais representativas na mensuração da atitude para com o computador, apresentando uma capacidade de explicar os valores observados de, respectivamente, $26 \%$ e $35 \%\left(R^{2}=0,26\right.$; 0,364). Além disso, observou-se que as variáveis ansiedade pelo uso do computador, gosto pelo computador e utilidade do computador no trabalho associam-se positiva e significativamente com a relação existente entre aluno e uso do computador. Diante dos achados, é importante destacar que atualmente o computador é visto como uma ferramenta imprescindível e costumeira no trabalho, e atitudes negativas em relação a sua aceitação podem influenciar significativamente no desempenho de funções no ambiente de negócios - fato que pode ter afetado significativamente os resultados.

Ademais, não foram encontradas relações significativas entre atitude do aluno em relação ao computador e sua aceitação da disciplina de Sistema de Informação Contábil. Acredita-se que esse comportamento pode ser desencadeado pelo fator cultural dos discentes, ou ainda, pelo forte hábito de uso dessa ferramenta. Em alternativa, sugere-se que pesquisas posteriores explorem a variável hábito para agregar valor às análises. Os resultados encontrados foram também contrários à relação proposta entre as variáveis de aceitação da disciplina de SIC pelos alunos e a percepção da utilidade dos SICs no local de trabalho. Incentiva-se que esse cenário seja explorado a fim de compreender quais são os motivos pelos quais os alunos não percebem uma possível aplicabilidade prática da disciplina de SIC no ambiente de trabalho, uma vez que pode ser, até mesmo, devido ao conteúdo programático de SIC. Conforme observado em Darayseh, Waples e Khaledi (2008), embora existam percepções positivas quanto à SIC na graduação, frequentemente o currículo dessa disciplina encontra-se em estágio defasado frente ao progresso da tecnologia no local de trabalho (DARAYSEH; WAPLES; KHALEDI, 2008). Acredita-se que esta lacuna pode ser mais bem investigada em pesquisas futuras.

Convém relatar as limitações desse estudo, sobretudo quanto ao campo analisado. Os resultados não podem ser generalizados para os alunos em outras universidades e faculdades, uma vez que envolveram o público de somente uma 
instituição de ensino e de uma região. Dessa forma, a aplicabilidade da metodologia em outras áreas nacionais com diferentes culturas e/ou nível de desenvolvimento (sudeste versus nordeste, por exemplo) pode apresentar resultados diferentes. Outrossim, acredita-se que o entendimento quanto ao conceito de SIC para os alunos é semelhante; no entanto, diferenças no método do ensino (por se tratar de diferentes turmas) poderiam influenciar os resultados relatados. Embora com limitações, este estudo fornece insights úteis para investigações de SIC no curso de Ciências Contábeis.

\section{REFERÊNCIAS}

AJZEN, I. The Theory of Planned Behavior. Organizational Behavior and Human Decision Processes, v. 50, p. 179-211, 1991.

AMERICAN ACCOUNTING ASSOCIATION (AAA). Computer Integration into the Accounting Curriculum, Accounting Education Series n. 8. (R.W. Ingram, Ed.), American Accounting Association, Sarasota, Florida, 1988.

Committee on Contemporary Approaches to Teaching Accounting Information Systems. Report of the AAA Committee on Contemporary Approaches to Teaching Accounting Information Systems, American Accounting Association, Sarasota, Florida, 1987.

AMERICAN INSTITUTE OF CERTIFIED PUBLIC ACCOUNTANTS (AICPA), 2004. Disponível em: <http://www.aicpa.org/ edu/candspe5.htm>. Acesso em: 14 jan. 2017.

AMERICAN INSTITUTE OF CERTIFIED PUBLIC ACCOUNTANTS (AICPA). Core Competency Framework, New York, USA, 2005. Disponível em: <http://ceae.aicpa.org/Resources>. Acesso em 15 fev. 2017.

BAGOZZI, R. P.; PHILLIPS, L. W. Representing and testing organizational theories: A Holistic Construal. Administrative Science Quarterly, v. 27, p. 459-489, 1982. 2011.

BAHAMONDEZ, V. E.; WINKLER, C.; SCHMIDT, A. Utilizing Capabilities for Mobile phones. ACM Press, New York, p. 935-944,

BIDO, D. S. Curso modelagem em equação estrutural com estimação através da estimação do modelo Partial Least Squares (PLS), 2008. Disponível em: <http://www.anpad.org.br/diversos/enanpad2012/minicurso_modelagem_equacoes_estruturais.pdf>. Acesso em: 20 dez. 2016.

BIDO, D. S.; SILVA, D.; SOUZA, C. A.; GODOY, A. S. Mensuração com indicadores formativos nas pesquisas em administração de empresas: como lidar com multicolinearidade entre eles? Administração: Ensino e Pesquisa, v. 11, n. 2, p. 45-269, 2010.

BORTHICK, F. A.; CLARK, R. L. Research on computing in accounting education: Opportunities and impediments. Issues in Accounting Education, p. 173-192, 1987.

CHIN, W. W.; NEWSTED, P. R. Structural equation modeling analysis with small samples using partial least squares. In: HOYLE, R. H. Statistical strategies for small sample research. Thousand Oaks, CA: Sage Publications, p. 307-341, 1999.

CONSELHO FEDERAL DE EDUCAÇÃO. Resolução n. 03, de 05 de outubro de 1992. Fixa os mínimos de conteúdo e duração do curso de graduação em ciências contábeis. Brasília: Ministério da Educação, out. 1992.

DAIGLE, R. J.; MORRIS, P. W. Fear Factor: The AIS Course and Non-Positive Attitudes towards Computers. Review of Business Information Systems (RBIS), v. 10, n. 1, p. 11-26, 2006.

DARAYSEH, M.; WAPLES, E.; KHALEDI, N. The Impact of the Acceleration of Information Technology on the Content of the Accounting Courses: The Case of GCC. Review of Business Information Systems (RBIS), v. 12, n. 3, 2008.

DUNN, C.; GRABSKI, S. The Effect of Field Dependence on Conceptual Modeling Performance. Advances in Accounting Information Systems, v. 6, p. 65-77, 1998.

EASON, K. D.; DAMODARAN, I. The Needs of the Commercial User, in Computer Skills and the User Interface (M.J. Coombs \& J.I. Atly Eds.), pp. 115-139, Academic Press, New York, 1981.

ELLIOT, R. K. Assurance Service Opportunities: Implications for Academia. Accounting Horizons, v. 11, n. 4, p. 61-74, 1997.

FÁVERO, L. P.; BELFIORE, P. P.; CHAN, B. L.; SILVA, F. L. Análise de dados: modelagem multivariada para tomada de decisões. Rio de Janeiro: Campus, 2009.

FERGUSON, C.; NEVELL, P. The Relationship Between Machine Enjoyment, Computer Attitude and Computer Usage: Some Further Refinements. Accounting and Finance, v. 36, n. 1, p. 113-125, 1996.

FORNELL, C.; LARCKER, D. F. Evaluating structural equation models with unobservable variables and measurement error. Journal of Marketing Research, v. 18, n. 1, p. 39-50, 1981.

GUJARATHI, M. Use of ERP software in accounting: A teaching note. Advances in Accounting Education, v. 7, p. 207-220, 2005.

HACKBARTH, G.; DOW, K.; JANVRIN, D.J. The influence of training environment on trainee expertise. AIS Educator Journal, v. 5. n. 1, p. 95-112, 2010.

HAIR JR., J. F.; BLACK, W. C.; BABIN, B. J.; ANDERSON, R. E.; TATHAM, R. L. Análise multivariada de dados. 6. ed. Porto Alegre: Bookman, 2009

HAIR JR., J. F.; HULT, T. M.; RINGLE, C. M.; SARSTEDT, M. A Primer on Partial Least Squares Structural Equation Modeling (PLS-SEM). Los Angeles: Sage, 2014.

HALL, M. The effect of comprehensive performance measurement systems on role clarity, psychological empowerment and managerial performance. Accounting, Organizations and Society, v. 33, n. 2/3, p. 141-163, 2008.

HARRIS, A. L. The Impact of the Introductory MIS Course on Students' Attitudes and Perceptions Towards microcomputers. The 
Journal of Computer Information Systems, v. 33, p. 33-41, 1992.

HOFFMAN, T. IT Auditors Coveted, Hard to Find. Computerworld, v. 38, n. 18, p. 1-16, 2004.

KACHELMEIER, S. J.; JONES, J. D.; KELLER, J. A. Evaluating the effectiveness of a computer-intensive learning aid for teaching pension accounting. Issues in Accounting Education, v. 7, p. 164-178, 1992.

LOYD, B. H.; LOYD, D. E. The reliability and validity of an instrument for the assessment of computer attitudes. Educational and psychological measurement, v. 45, n. 4, p. 903-908, 1985.

MACUR, K. M. The Curricular Content of Accounting Information Systems. Review of Business Information Systems (RBIS), v. 2, n. 3, p. 11-20, 2011.

MAHONEY, L. S.; WELCH, J. K. Teaching accounting information systems using personal computer movies. Advances in Accounting Education, v. 4, p. 119-138, 2002.

MINCH, R. P.; RAY, N. M. Alienation and computer user attitudes. Proceedings of the Seventh International Conference on Information Systems, p. 168-180, 1986

MORRIS, P. W.; DAIGLE, R. J. Student Attitudes Towards Computers Before and After Taking an Introductory AIS Course. Journal of Business \& Economics Research, v. 2, n. 7, p. 19-36, 2004.

OMAR, M. H. Impact of the MIS Course on Business Students' Attitudes to Computers and on Their Understanding of Information Systems Concepts. The Journal of Computer Information Systems. v. 32, n. 1, p. 36-39, 1991.

PRADO, A. G. S.; GUEDES, S. M. L. C.; PAIVA, S. B. P. Um estudo comparativo das ementas da disciplina sistemas de informações contábeis. In: XI Encontro de Iniciação à Docência. Universidade Federal da Paraíba, 2008. Anais... Disponível em: <http://www.prac. ufpb.br/anais/xenex xienid/xi enid/monitoriapet/ANAIS/Area4/4CCSADFCMT02.pdf>. Acesso em: 23 de junho de 2017. 1991.

RAVEL, V. Perspectives on Students' Teaching Evaluations of AIS Courses. The Journal of Information Systems, v. 5, n. 2, p. 62-72,

SHNEIDERMAN, B. Human Factors Experiments in Designing Interactive Systems. Computer, v. 12, n. 9, p. 24, 1979.

STANLEY, T.; EDWARDS, P. Interactive multimedia teaching of Accounting Information System (AIS) cycles: Student perceptions and views. Journal of Accounting Education, n. 23, v. 1, p. 21-46, 2005.

TAN, T.; GODFREY, A. Information Technology Knowledge and Skills Required by Accounting Graduates in New Zealand. MILLER, P.; DREYER, W. Doctoral research in management and business in New Zealand. Southern Cross University Press: Lismore, NSW, 2012.

TOGO, D. F. Acquiring an overview of accounting as an information system for planning, control, and reporting: A spreadsheet approach. The Journal of Information Systems, v. 5, p. 73-77, 1991.

TOGO, D. F.; MCNAMEE, A. H. Computer Integration into the Accounting Curriculum: Learning Benefits, Problems, and Guidelines. Journal of Accounting Education, v. 13, n. 2, p. 149-158, 1995.

TOGO, D. F; YUTHAS, K. Role Playing and Cooperative Learning in The AIS Course. Review of Business Information Systems (RBIS), n. 5, v. 2, p. 63-70, 2011.

TORKZAHEH, G.; KOUFTEROS, X. Computer User Training and Attitudes: A Study of Business Undergraduates. Behaviour \& Information Technology, v. 12, n. 5, p. 284-292, 1993.

WELI. Accounting Students Attitude towards Computer, the Acceptance of the Accounting Information System's Course and Teaching Method. Procedia - Social and Behavioral Sciences, v. 172, p. 18-25, 2015.

R: WONG, H.; WONG, R. Students' Perceptions on Studying Accounting Information System Course. International Journal of Business Administration, v. 8, n. 2, 2017.

WU, F. H. (1986). Spreadsheet software: A tool for teaching managerial (cost) and financial accounting. The Journal of Information Systems, v. 1, p. 121-136, 1986.

. Teaching accounting information systems: A synthesis. Issues in Accounting Education, v. 1, n. 1, p. 132-145, 1983. 


\section{APÊNDICE A. INSTRUMENTO DE PESQUISA}

Bloco A. Ansiedade com Computador = CA

CA1: Os computadores não me assustam;

CA2: Trabalhar com um computador me estressa muito;

CA3: Computadores me fazem sentir desconfortável;

CA4: Computadores me fazem sentir inquieto e confuso.

\section{Bloco B. Gosto por Computador (CL)}

CL1: Eu gostaria de trabalhar com computadores;

CL2: Eu não entendo como algumas pessoas podem gastar tanto tempo trabalhando com computadores e parecem se divertir;

CL3: Uma vez que eu começar a trabalhar com computador, dificilmente iria conseguir parar de usá-lo;

CL4: Eu não gosto de falar com os outros sobre computadores.

\section{Bloco C. Utilidade Percebida no uso do computador no local de trabalho (TCU)}

CUW1: Meu conhecimento em computadores me ajuda a conseguir um emprego melhor;

CUW2: Meu conhecimento em computadores me dá a vantagem sobre os outros permitindo-me para conseguir um emprego mais rápido do que os outros;

CUW3: Meu conhecimento em computadores ajuda-me a ser promovido mais rápido;

CUW4: Meu conhecimento em computadores torna a minha vida de trabalho mais fácil;

CUW5: Meu conhecimento em computadores faz minha comunicação mais fácil e mais rápida com os outros;

CUW6: Meu conhecimento em computadores ajuda-me a fazer o meu trabalho atual melhor;

CUW7: Eu preciso do computador fora do meu ambiente de trabalho;

CUW8: Eu estou sempre em necessidade de formação de computador para ser atualizado em fazer o meu trabalho (preciso estar atualizado sempre com computadores para realizar meu trabalho).

\section{Bloco D. Importância Percebida do Computador no local de Trabalho (CIW)}

CIW1: É importante para o meu trabalho que eu saiba utilizar bases de dados;

CIW2: É importante saber como usar planilhas no meu trabalho;

CIW3: É importante estudar como usar pacotes e programas de computador nos anos escolares;

CIW4: É importante estudar como usar computadores em auditoria;

CIW5: É muito importante compreender o sistema de computador que eu uso no meu trabalho.

\section{Bloco E. Utilidade Percebida do Sistema de Informação Contábil no Local de Trabalho (AUW)}

AUW1: O Sistema de Informação Contábil e outros cursos que fiz durante meus anos de escola foram suficientes para me ajudar a fazer outras coisas fora do meu ambiente de trabalho;

AUW2: As disciplinas que fiz durante os meus anos escolares discutiam as necessidades de empresas e instituições voltadas para informação (bases de dados) e planejamento;

AUW3: As disciplinas que fiz durante meus anos de escola discutiram os problemas que enfrentam as empresas e instituições em matéria de controle da informação;

AUW4: As disciplinas que fiz durante meus anos de escola discutiram os problemas que enfrentam empresas e instituições com relação ao uso e distribuição de bases de dados;

AUW5: Há uma forte relação entre as disciplinas que fiz na escola e a base de dados utilizada no meu trabalho.

Bloco F. Aceitação de Sistemas de Informação Contábil no Curso (PNM)

Introdução à AIS: Perspectiva Contador

Introdução ao processamento de transações

Ética, Fraude e Controle Interno

Computador, Fraude e Abuso Técnicas 
Técnica Documentação: Diagrama de Fluxo de Dados, Fluxograma / UML

Diagrama, Diagramas de Relação de Entidade

O ciclo de receitas: As vendas a dinheiro Coleções

O ciclo de despesa: Comprando a Caixa, Desembolsos

O Ciclo de Gestão de Recursos Humanos e Folha de Pagamento

Procedimentos de Imobilização

O Ciclo de Conversão / o ciclo de produção

Relato Financeiro e Controle de Gestão / Livro razão geral e sistema de comunicação

Sistemas de Gerenciamento de Banco de Dados

A Abordagem Recursos-agente-evento (REA) para o processo de modelo de negócios

Enterprise Resource Planning Systems (ERP)

Sistemas de comércio eletrônico

Gerenciando o Ciclo de Vida de Desenvolvimento de Sistemas / Construir, entregar e manter sistemas de Projeto

Controles de TI: Sarbanes-Oxley e Governança de TI

Controles de TI: Segurança e Acesso

Controles de TI: Desenvolvimento de Sistemas, Alterações de programas, Controles de Aplicação

Sistemas de informação de Auditoria computadorizados

\section{Bloco G. Métodos de Ensino}

MP1: Conferências

MP2: Palestras (aulas) usando o PowerPoint

MP3: Grupo de Discussão

MP4: Discussão de textos em classe

MP5: Perguntas e respostas

MP6: Aulas e Vídeos

MP7: Dando um exemplo de Programa de Computador

MP8: Utilizar o e-learning (o ensino eletrônico, EAD)

MP9: Usando Internet

MP10: Estudo de campo 